\title{
SURFACE MICROMACHINED AND INTEGRATED CAPACITIVE SENSORS FOR MICROFLUIDIC APPLICATIONS
}

\author{
Jason Shih, Jun Xie, Yu-Chong Tai \\ Department of Electrical Engineering \\ California Institute of Technology, CA 91125, US
}

Tel.: (626) 395-1364, Fax: (626) 584-9104, email: jshih@touch.caltech.edu

\begin{abstract}
We have demonstrated an entire series of capacitive sensors using a multi-layer parylene/photoresist surface micromachining technology. The developed sensors are designed for total integration into parylene-based microfluidic systems for real-time system monitoring. Sensors have been demonstrated for the following applications: 1) in-line pressure sensing (range: 0 - $35 \mathrm{kPa}$, resolution: $0.03 \mathrm{kPa}$ ); 2) liquid front position and/or volumetric measurements (range: $0->50$ $\mathrm{pL}$, resolution: $<5 \mathrm{pL}$ ); and 3) dielectric measurements, which can be used to deduce fluid properties such as liquid composition. The reported sensor technology demonstrates versatility, high sensitivity, small footprints, and easy integration.
\end{abstract}

\section{INTRODUCTION}

The total integration of many microfluidic components has been a long sought goal in the microfluidics community. The incompatibility of different processes renders many demonstrated devices as stand-alone. The multilayer parylene/photoresist surface micromachining technology utilized here, allows for the integration of different microfluidic components onto a single chip. Previously demonstrated microfluidic devices utilizing this technology include pumps, check valves [1], and a mass flow controller [2]. In addition, microfluidic systems such as an electrolysis-based system for electrospray ionization mass spectroscopy (ESI-MS) have been developed [3]. Because of the common technology used to create these devices, total integration is possible. But with the exception of a hot-wire type thermal flow sensor used in the mass flow controller [2], a sensor technology had yet to be developed. Sensors are necessary for feedback control and for the monitoring of the microfluidic system. Only then can the goal of autonomous lab-on-a-chip systems be realized. The presented capacitance based sensors fill this need.

Previously, capacitive sensors have been applied to microfluidic applications in the form of pressure sensors and flow sensors based on a differential pressure principle [6]. Capacitive sensing has also been utilized in macrofluidic applications such as fluid level sensing, determination of ion concentration [7], and for measuring the makeup of mixtures (eg. oil and water). The presented sensors exhibit all the functionality of the previously mentioned sensors, but with the added flexibility and ease of integration of our developed parylene/photoresist surface-micromachining technology. a

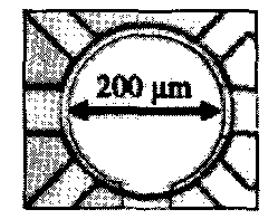

b

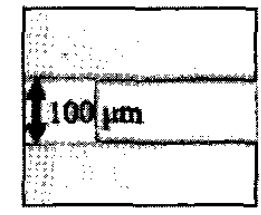

c

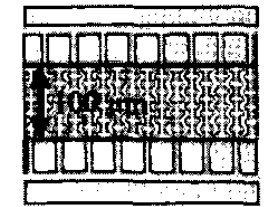

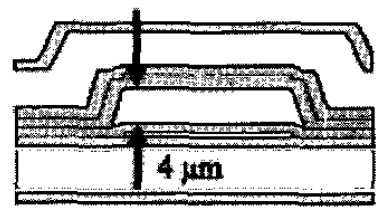
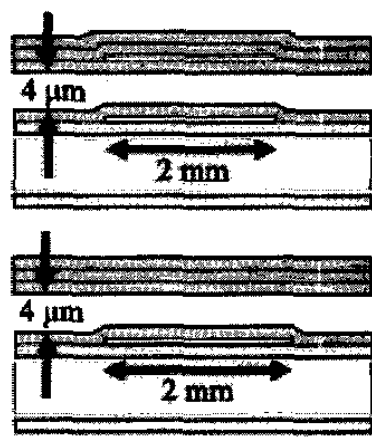

Figure 1: Micrographs and Cross Sectional Views (Oriented Along Channel) of the Fabricated Sensors, a. Pressure Sensor, b. Parallel Plate Configuration, c. Interdigitated Configuration

\section{- DESIGN}

Three different sensor configurations are investigated. These include a membrane based pressure sensor; a parallel plate configuration for dielectric measurements; and an interdigitated configuration for use in volumetric and dielectric measurements.

The pressure sensor is realized by creating a cavity beneath the channel. The upper electrode is sandwiched between the floor of the channel and another parylene layer, forming a $200 \mu \mathrm{m}$ diameter composite membrane. The bottom electrode is fixed on the substrate. The fabricated device and schematic cross section are shown in figure 1a. Changes in the local channel pressure will cause a deflection of the membrane/electrode. The capacitance therefore varies as a function of the local pressure in the channel. The air gap is not sealed, making this a differential pressure sensor. Since the capacitive element is completely underneath the channel, the pressure reading is independent of the fluid being carried.

The parallel plate electrode configuration consists of two electrodes, one placed in the roof of the channel and the other placed in the floor of the channel. This configuration is shown in figure $1 \mathrm{~b}$. The channel height is $4 \mu \mathrm{m}$ and the width of the channel and electrodes is $100 \mu \mathrm{m}$. Each electrode is $2 \mathrm{~mm}$ long. Both the

\section{TRANSDUCERS ' 03}

The 12th International Conference on Solid State Sensors, Actuators and Microsystems, Boston, June 8-12, 2003 
electrodes are electrically isolated from the fluid with a 1 $\mu \mathrm{m}$ parylene layer. Changes in the dielectric constant of the channel fluid will register as a capacitance change. Properties such as the mixing ratio of two pure liquids can be determined through appropriate calibration since the effective dielectric constant is a function of the mixing ratio. Also, pure liquids can be identified provided they have different dielectric constants. Besides determining fluid properties; such a sensor can be used to easily determine whether a particular channel is filled.

The interdigitated electrode configuration consists of two interlocking comb-shaped electrodes as seen in figure 1c. Both electrodes are placed directly beneath the floor of the channel. The spacing between adjacent fingers is $15 \mu \mathrm{m}$ and the electrodes overlap by $100 \mu \mathrm{m}$, also the width of the channel. The channel height is $4 \mu \mathrm{m}$ and the total sensor length is $2 \mathrm{~mm}$. Again, the electrodes are insulated from the channel with a $1 \mu \mathrm{m}$ parylene layer. The fringing electric field reaches into the channel so changes in the dielectric constant of the channel fluid are registered as a capacitance change. Besides using the interdigitated configuration for the same applications mentioned for the parallel plate configuration, it can also be used to monitor fluid front position. As a column of liquid flows into an empty channel, a predictable increase in capacitance is seen. This capacitance is a function of the fluid front position, which gives a direct measurement of volume.

Compared to the parallel plate configuration, the interdigitated configuration is simpler from a fabrication standpoint since only one layer of metal is needed. Also, the interdigitated configuration does not obscure the channel, therefore making it more desirable for calibration purposes. But, since the interdigitated sensor exploits the fringing electric fields, a weaker signal is expected.

\section{FABRICATION}

The sensors are fabricated using a multilayer parylene/photoresist surface micromachining technology. Four parylene layers and two sacrificial photoresist layers are used in the fabrication of these sensors. Because of the way these sensors are designed, they are automatically integrated into microchannels. $\mathrm{Cr} / \mathrm{Au}$ is used for the various electrodes and a sputtered silicon layer is used to ensure a freestanding pressure sensor membrane, a method described in [4].

The full fabrication process flow is shown in figure 2. The process begins with the oxidation of a silicon wafer. The oxide is patterned and DRIE used to etch the backside fluidic ports that will be used to introduce liquids. A Cr/Au layer $(100 \AA / 3000 \AA)$ is deposited and patterned. This metal layer defines the bottom electrodes of the pressure sensor and parallel plate configuration. This layer is also used to define both electrodes in the interdigitated configuration. After a 1 $\mu \mathrm{m}$ parylene layer is deposited, a $4 \mu \mathrm{m}$ photoresist sacrificial layer defines the capacitive air gap in the pressure sensor. This sacrificial layer also defines the

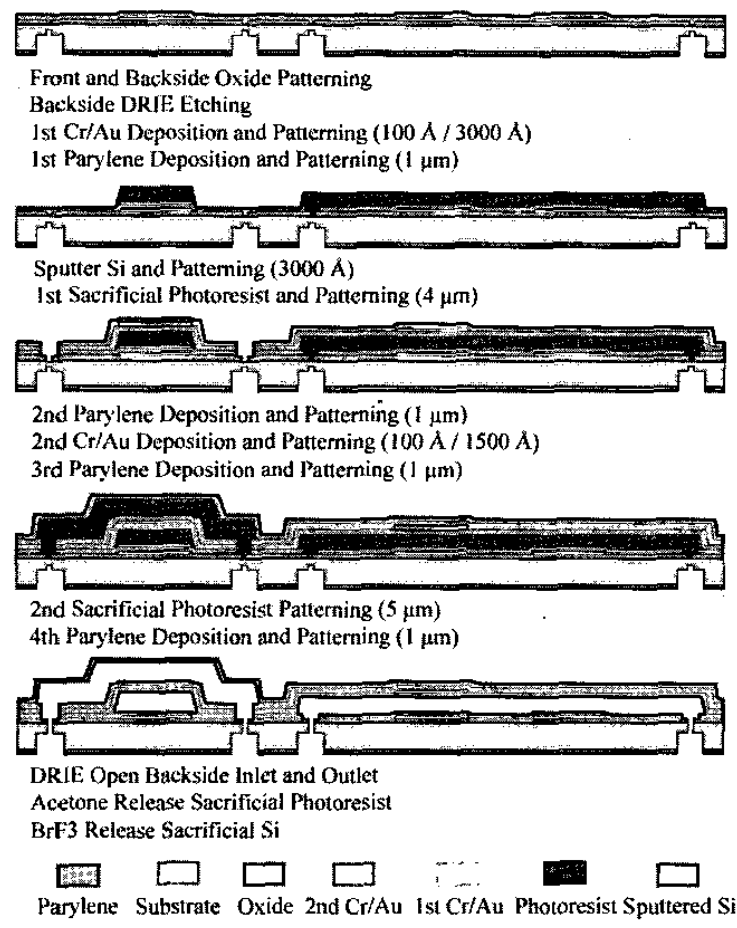

Figure 2: Full Process Flow

microchannel in which the parallel plate and interdigitated sensors are integrated. After another $1 \mu \mathrm{m}$ parylene layer, a second $\mathrm{Cr} / \mathrm{Au}$ layer $(100 \AA / 1500 \AA)$ layer is deposited and patterned to produce the top electrodes of the pressure sensor and parallel plate configuration. A third $1 \mu \mathrm{m}$ parylene layer is deposited and a $5 \mu \mathrm{m}$ photoresist sacrificial layer is deposited and patterned to define the channel above the pressure sensor. Finally, DRIE is used to etch the fluidic ports completely through the wafer and the photoresist is released by acetone. Upon drying of the acetone, the pressure sensor membrane will often stick down because of stiction. Gas phase $\mathrm{BrF}_{3}$ is used to etch away the sputtered silicon and produce a freestanding membrane.

\section{RESULTS AND DISCUSSION}

\section{Pressure Sensor}

The pressure sensor is calibrated using a regulated pressure source. Pressure is applied to both ends of the channel simultaneously by means of a custombuilt Plexiglas jig, thus ensuring the channel pressure is at the desired value. The pressure is varied and the changing capacitance is measured. Capacitances are measured using a commercially available IC (Microsensors UCR MS3110). Calibrations of the pressure sensor are done using both water and air as the channel fluid. The results are shown in figure 3 . The sensor resolution is estimated to be $0.03 \mathrm{kPa}$ and the accuracy to be $1.0 \mathrm{kPa}$. 


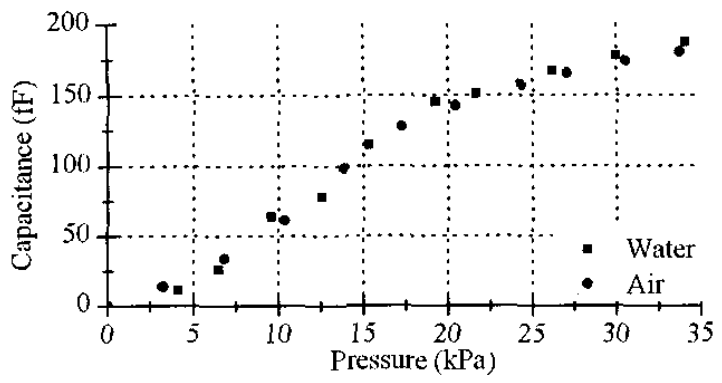

Figure 3: Pressure Sensor Calibration with Air and Water

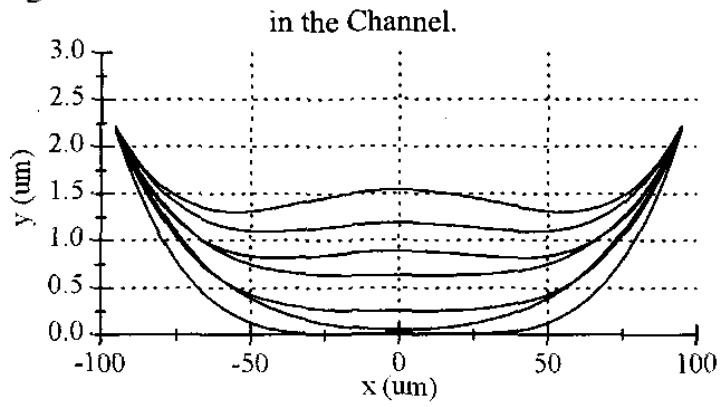

Figure 4: The Membrane Profile at Different Pressures

(From Top to Bottom $(\mathrm{kPa})$ : 0.00, 3.45, 7.03, 10.62,

$13.65,17.23,24.26)$

The response of the sensor shows a roughly linear increase in capacitance at pressures below $20 \mathrm{kPa}$. At higher pressures, the sensitivity decreases and the capacitance begins to plateau. This is likely caused by the membrane bottoming out. To investigate the pressure sensor behavior more thoroughly, the deflection of the top electrode is measured at various applied pressures using a WYKO NT2000 Optical Profiler. The membrane profile at different pressures is shown in Figure 4.

The initial membrane is not flat, as would be expected. Instead, the membrane exhibits a large downward deflection with the center of the membrane slightly bowed upward. This signals that the membrane has been distorted during the fabrication process. Most likely this deformation occurred during the drying process. After photoresist dissolution, stiction occurs betweens the pressure membrane and the floor of the capacitive air gap. The forces exerted on the membrane during drying may have been high enough to permanently deform the membrane.

In the fabrication of the pressure sensor, a $4 \mu \mathrm{m}$ photoresist layer is used to define the air gap. Our measurements, as seen in figure 4 , indicate a total deflection of only $1.5 \mu \mathrm{m}$. This discrepancy is once again due to the distortion of the membrane. This distortion effectively decreases the pressure range of this sensor. Our measurements also indicate that the membrane bottoms out at approximately $20 \mathrm{kPa}$. This coincides precisely with the measured sensor response.

A test of the pressure sensor is done by applying pressure to one end of the microchannel and leaving the other end open. The pressure sensor is located in the center of this channel. By varying the applied pressure and at the same time recording the pressure sensor output and flow rate, we are able to demonstrate the capability of this sensor to monitor the local pressure inside the channel. This also functions as flow sensor based on a differential-pressure principle.

The applied pressure and the measured channel pressure are shown in figure 5 , and are both related to the corresponding flow rate. The flow rate vs. applied pressure data is fit using equation 2 , as found in [5].

$$
Q=\frac{w h_{o}^{3} \Delta P}{12 v L}(1+2 \alpha \Delta P)
$$

The $1+2 \alpha \Delta \mathrm{P}$ accounts for bulging of the microchannel. The measured data is fit assuming an air viscosity of $1.73 \mathrm{e}-5 \mathrm{~kg} / \mathrm{s}-\mathrm{m}$. By fitting our data, $\alpha$ is found to be $0.0065 / \mathrm{kPa}$. The measured pressure in the center of the channel is found to be precisely 0.41 of the applied pressure. The line corresponding to 0.41 of the applied pressure is shown in figure 5. A similar test is done using ethanol as the channel fluid. The results are shown in figure 6 . The pressure at the center of the channel is found to be approximately 0.46 of the applied pressure in this test.

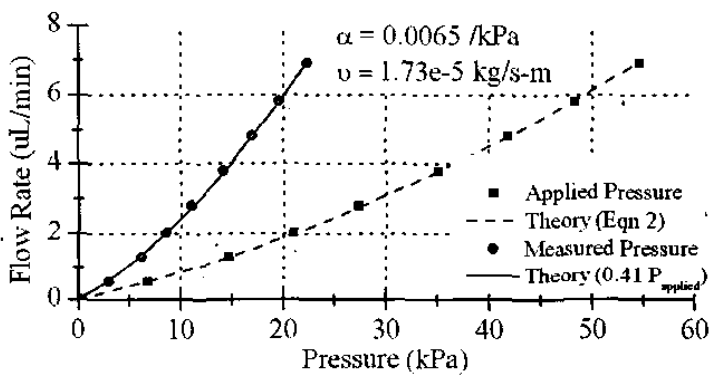

Figure 5: Monitoring of Pressure in the Channel (Air as the Fluid)

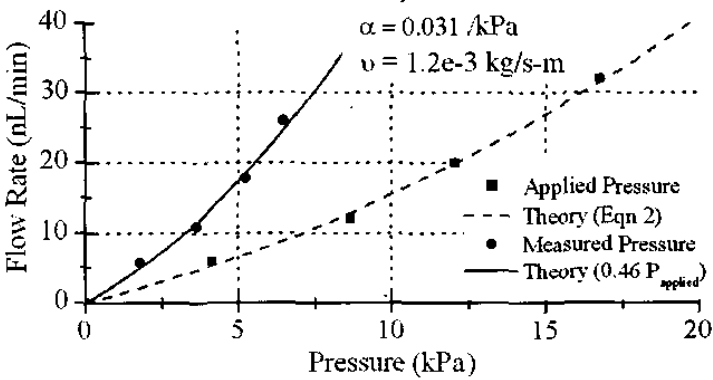

Figure 6: Monitoring of Pressure in the Channel (Ethanol as the Fluid)

\section{Volumetric Sensor}

The volumetric sensor is calibrated by slowly introducing water into the interdigitated sensor. The introduction of the liquid is captured on video and correlated with the capacitance measurements. The measurements shown in figure 7 correspond to the

\section{TRANSDUCERS '03}

The 12th International Conference on Solid State Sensors, Actuators and Microsystems, Boston, June 8-12, 2003 
capacitance as the fluid front passes over each successive finger. Since the cross section of the channel is fixed, this fluid front position corresponds directly to a volume. The spacing between each finger is $15 \mu \mathrm{m}$ which corresponds to $6 \mathrm{pL}$. The capacitance change as a function of volume is shown in figure 7 .

The sensor shows very high linearity in this short section of the sensor. A resolution of $<5 \mathrm{pL}$ should be attainable.

\section{Dielectric Measurements}

One simple use of the parallel plate and interdigitated sensors is to detect the presence of a fluid in a channel. For example, the capacitance of our sensor would increase significantly if there is water in the channel as opposed to air. For the interdigitated sensor the increase in capacitance is measured to be $1.11 \mathrm{pF}$ and for the parallel plate configuration it is measured to be $3.38 \mathrm{pF}$. Since different fluids have different dielectric constants, it is also possible to distinguish a large variety of fluids, provided their dielectric constants are different.

The composition of a mixture of liquids can also be determined if the two liquids mix predictably. Since the effective dielectric constant is a function of the distribution of the two components as well as the individual dielectric constants, the distribution must be predictable for this method to work. A proof of concept is done using mixtures of Isopropyl Alcohol and water. The measured interdigitated capacitance increases for varying mixtures is shown in figure 8 . The capacitance is expressed as an increase over an empty channel.

\section{CONCLUSION}

The demonstrated sensors show great promise for microfluidic applications because of their high sensitivity and easy integration. The versatility of the parylene/photoresist surface micromachining technology allows for the integration of these sensors with other demonstrated devices for the creation of entire microfluidic systems.

\section{ACKNOWLEDGEMENTS}

This work is supported in part by NSF ERC Center at Caltech (Grant No. EEC-9402726), NIH (Grant No. 5R01RR06217-10), and DARPA/MTO Bioflips Program.

\section{REFERENCES}

[1] J. Xie, X. Yang, X.Q. Wang, ,Y.C. Tai, "Surface Micromachined Leakage Proof Parylene Check Valve." 14th IEEE International Conference on Micro Electro Mechanical Systems (MEMS '01). 2001. pg. 539-542.

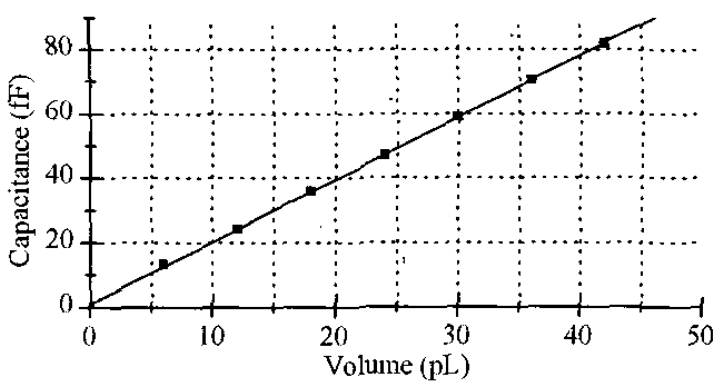

Figure 7: Capacitance as a Function of Volume for the Interdigitated Sensor

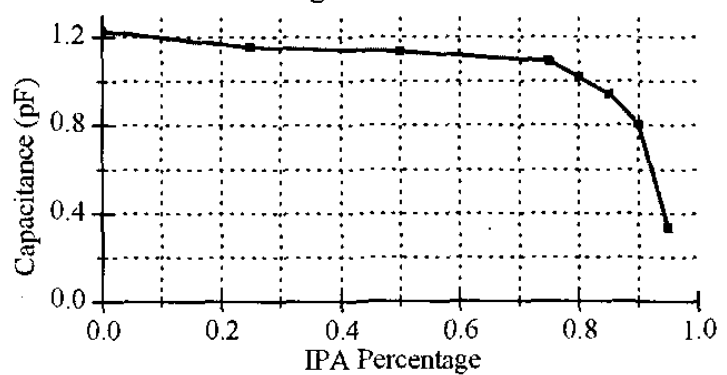

Figure 8: Capacitance as a Function of IPA Percentage for the Interdigitated Sensor

[2] J. Xie, J. Shih, Y.C. Tai. "Integrated SurfaceMicromachined Mass Flow Controller." 16th IEEE International Conference on Micro Electro Mechanical Systems (MEMS '03). 2003. pg. 20-23.

[3] J. Xie, Q. He, Y.C. Tai, J. Liu, T. Lee. "ElectrolysisBased On-Chip Dispensing System for ESI-MS." 16th IEEE International Conference on Micro Electro Mechanical Systems (MEMS '03). 2003. pg. 443-446.

[4] T.J. Yao, X. Yang, Y.C. Tai. "BrF 3 Dry Release Technologies for Large Freestanding Parylene MEMS." International Conference on Solid-State Sensors and Actuators (Transducers '01). 2001. pg. 652-655.

[5] S. Wu, J. Mai, Y. Zohar, Y.C. Tai, C.M. Ho. "A Suspended microchannel with Integrated Temperature Sensors for High-Pressure Flow Studies." 11th IEEE International Workshop on Micro Electro Mechanical Systems (MEMS '98). 1998. pg. 87-92.

[6] R.E. Oosterbroek, T.S.J. Lammerink, J.W. Berenschot, A. van den Berg, Elwenspoek. "Designing, Realization And Characterization of A Novel Capacitive Pressure/Flow Sensor." International Conference on Solid-State Sensors and Actuators (Transducers '97). 1997. pg.151-154.

[7] K. Shida, A.B.M. Ismail. "Measurement of Concentration of Electrolytic Solution Based on Novel Non-Contact Multi-Sensing Techniques." IEEE Instrumentation and Measurement Technology Conference, 1999 (IMTC'99). 1999. pg. 1372- 1376. 